\title{
A brief overview of animal symptom checkers
}

\author{
Jelle Stans* \\ Institute for Globally Distributed Open Research and Education (IGDORE). Independent, Beringen, Belgium
}

\begin{abstract}
Symptom checkers are tools that provide health information, including possible conditions, after entering one or more symptoms. Some symptom checkers also provide advice on how urgent medical attention should be sought. In addition to human symptom checkers, several tools are also available to check the symptoms of animals and provide veterinary triage advice. Unlike in human symptom checkers, this widespread availability has not lead to investigations of these tools. Indeed, little to no peer-reviewed research has been published regarding animal symptom checkers. This paper aims to describe some examples of animal symptom checkers. In addition, the proposals for future research are formulated by translating knowledge obtained from research into human symptom checkers.
\end{abstract}

Keywords: Animal, Online, Pet, Symptom checker.

\section{Introduction}

Symptom checkers are tools that provide health information, including possible conditions, after entering one or more symptoms. Some symptom checkers also provide advice on how urgent medical attention should be sought (Semigran et al., 2016; Morita et al., 2017; Berry, 2018).With 80.000.000 households in the European Union owning a pet (Fediaf report, 2018) and 75\% of web users looking for (human) health information online (Starcevic and Berle, 2013), there probably is also a demand for these symptom checkers for animals. Three examples are described in this paper. The PetMD symptom checker was selected because it is the first search result for a variety of combinations of keywords (e.g., "symptom checker dog" and "symptom checker cat") relating to animal symptom checkers with the search engine Google. The VetHelpDirect symptom checker is also described because it is available from a variety of external websites. It is also in the top results for the abovementioned keyword searches. In addition, it is an alternative for species that the PetMD symptom checker does not cover. The symptom analysis tool of the Petriage application for smartphones was selected as the final symptom checker because it is available for free. Despite the research performed in the area of human symptom checkers, the body of evidence regarding animal symptom checkers is very limited. A search of the PubMed, PubMed Central, and Web of Science databases did not yield any peer-reviewed studies focusing specifically on symptom checkers in animals. A manual search of the index of several veterinary and e-Health journals also did not result in literature regarding the subject. This paper aims to describe some examples of animal symptom checkers. In addition, the proposals for future research are formulated by translating knowledge obtained from research into human symptom checkers.

Description of examples of animal symptom checkers

The PetMD symptom checker is available for both cats and dogs. After selecting the animal of choice, the user is asked to select an area of the body, after which the tool presents a possible list of symptoms related to this area. The user is also able to select "behavior" and mark symptoms such as aggression and anxiety. Depending on the specific (combination of) symptom(s), the tool will provide several articles about possible conditions. These articles cover the condition in the species, the associated symptoms, causes, diagnosis, treatment, management, and prevention.The VetHelpDirect symptom checker, which is also available through several external sources, is available for a broad range of animals including cats, dogs, and rabbits. After choosing the animal, the user is asked to select a symptom. Subsequently, the tool asks several specific questions to obtain more information about the symptom and condition of the animal. Afterward, a triage advice is provided, as well as suggestions for first aid and possible conditions. The Petriage application is available for both iOS- and Android-operating smartphones and also includes a symptom analysis tool. After creating a profile for your pet, some basic information such as species (cat or dog), breed, age, gender, and weight are requested. After the completion of the profile, you can start a symptom analysis by manually entering a symptom through a search function. Subsequently, the application asks information about vital signs such as the pet's temperature and respiratory rate. More specific information about the symptoms is also requested such as when it started, how often it happened already, and whether it is worsening. After completing all the information, the application provides a triage advice. 
At this time, no animal symptom checkers were identified which provided the user with a ranking of conditions according to their likelihood based on the symptoms entered. Such symptom checkers are currently available for human conditions.

\section{Advantages, disadvantages, and limitations of animal symptom checkers}

A first advantage of animal symptom checkers is that they may urge owners to seek veterinary care when required or reduce unnecessary visits to the veterinary clinic. On the other hand, if the triage advice is inaccurate, owners may postpone going to the veterinarian when urgent medical attention is required. A study by Semigran et al. (2015) showed that human symptom checkers tend to avoid risk and direct users to seek medical care when this is not required. Whether this is also the case for symptom checkers used for pets, is not clearly established yet, and warrants further research. Questionnaires could be provided to animal owners seeking veterinary attention to assess whether they prechecked their animal's symptoms using a symptom checker and whether this influenced their decision to seek medical help and in which way. To capture information about owners not seeking veterinary support after using a symptom checker, an invitation to participate in an online survey could be send to users of an online symptom checker.

Symptom checkers may also provide advice to owners who may otherwise not seek, cannot afford, or do not have access to veterinary care. It should always be mentioned, however, that symptom checkers cannot replace veterinary care and that adequate veterinary help should always be sought when animal health problems are possible or suspected.

Like in humans, some diagnoses are more common in certain animals than in others. A study by Semigran et al. (2015) found that human symptom checkers performed less well in identifying uncommon conditions than common ones. The triage advice for uncommon conditions was, however, more often correct (Semigran et al., 2015). Furthermore, in animals, some conditions may be better understood in one species than in another. This means that the performance of a symptom checker for a specific condition may differ between animals and that, within the same species, a symptom checker may perform better in detecting one condition than another. This may support the idea of developing separate algorithms and symptom checkers for one type of animal instead of general tools covering different species. These tools can then be tweaked to the specific species they are designed for.

Another disadvantage or limitation of symptom checkers in animals could be that, by definition, the symptoms entered into such a tool may be more often incomplete or more inaccurate than those entered in human symptom checkers. It may be more difficult for a person to assess symptoms in an animal than it is to describe their own symptoms. It can also be difficult to find words to describe specific symptoms if symptom checkers do not use lay language.Finally, a possible advantage of using animal symptom checkers may be to reassure owners and reduce the amount of worrying by providing information. On the other hand, it has been shown that accessing health information online can worsen health anxiety (Starcevic and Berle, 2013; Starcevic, 2017). To what degree using animal symptom checkers has either of these effects and warrants future research. An exploratory study investigating the goal of people using these symptom checkers and the effect that they have on the mental health of animal owners could provide important insights. Furthermore, the effect of this influence has on the decision whether and what type of veterinary care to look for is an interesting research topic.

\section{Accuracy of information provided by animal symptom checkers}

The diagnostic accuracy and performance of symptom checkers for humans have been tested in a variety of specific conditions, ranging from ophthalmic conditions (Shen et al., 2019) to knee pain (Bisson et al., 2016). The accuracy of these symptom checkers has also been compared to these physicians (Semigran et al., 2016). In another study, Semigran et al. (2015) also investigated how often symptom checkers provided correct triage advice for certain conditions.

In several of these studies, it was shown that symptom checkers underperform as compared to physicians (Semigran et al., 2016; Berry et al., 2019). Morita et al. (2017) stated that symptom checkers can improve their diagnostic accuracy based on appropriate feedback.

There has been little to no peer-reviewed research regarding the diagnostic accuracy of veterinary symptom checkers. This lack of research could be partly due to the fact that, unlike some human symptom checkers, the current animal symptom checkers do not attach the likelihood to the possible conditions that are mentioned. An audit study as performed by Semigran et al. (2015) in humans, using standardized vignettes, may provide some preliminary insights as to how often the correct diagnosis is mentioned in the list of possible conditions.

Since no animal symptom checkers provide a "most likely" condition, performing research to compare the accuracy between the tool and a veterinarian is currently difficult. This lack of research may hamper the "appropriate feedback" mentioned above and thus lead to a slowdown in the improvement and accuracy of animal symptom checkers. The development of an algorithm that can list the likelihood of conditions based on symptoms entered could be the start of research into the diagnostic accuracy. If the diagnostic accuracy of this kind of algorithms is shown to be limited, it should be considered to first optimize the algorithm before releasing it to the public for use in symptom checkers and for use outside of a research environment. 


\section{Conclusion}

Despite the availability of animal symptom checkers, there has been little to no peer-reviewed literature published about the subject. Studies should be carried out on the accuracy, usage, and other aspects of these tools. To perform these studies, researchers can base themselves on the body of literature that is available for human symptom checkers.

\section{Conflict of interest}

The author declares that there is no conflict of interest.

\section{References}

Berry, A.C. 2018. Online symptom checker applications: syndromic surveillance for international health. Ochsner. J. 18(4), 298-299.

Berry, A.C., Cash, B.D., Wang, B., Mulekar, M.S., Van Haneghan, A.B., Yuquimpo, K., Swaney, A., Marshall, M.C. and Green, W.K. 2019. Online symptom checker diagnostic and triage accuracy for HIV and hepatitis C. Epidemiol. Infect. 147, e104. doi:10.1017/S0950268819000268

Bisson, L.J., Komm, J.T., Bernas, G.A., Fineberg, M.S., Marzo, J.M., Rauh, M.A., Smolinski, R.J. and Wind, W.M. 2016. How accurate are patients at diagnosing the cause of their knee pain with the help of a web-based symptom checker? Orthop. J. Sports Med. 4(2), 2325967116630286. doi: $10.1177 / 2325967116630286$
Fediaf report. 2018. "European facts \& figures". Available via http://www.fediaf.org/images/ FEDIAF_Facts_and_Figures_2018_ONLINE_ final.pdf

Morita, T., Rahman, A., Hasegawa, T., Ozaki, A. and Tanimoto, T. 2017. The Potential Possibility of Symptom Checker. Int. J. Health Policy Manag. 6(10), 615-616.

Semigran, H.L., Levine, D.M., Nundy, S. and Mehrotra, A. 2016. Comparison of physician and computer diagnostic accuracy. JAMA Intern. Med. 176(12), 1860-1861.

Semigran, H.L., Linder, J.A., Gidengil, C. and Mehrotra, A. 2015. Evaluation of symptom checkers for self diagnosis and triage: audit study. BMJ, 351, h3480. doi:10.1136/bmj.h3480

Shen, C., Nguyen, M., Gregor, A., Isaza, G. and Beattie, A. 2019. Accuracy of a popular online symptom checker for ophthalmic diagnoses. JAMA Ophthalmol. 137(6), 690-692.

Starcevic, V. 2017. Cyberchondria: challenges of problematic online searches for health-related information. Psychother. Psychosom. 86(3), 129-133.

Starcevic, V. and Berle, D. 2013. Cyberchondria: towards a better understanding of excessive health-related Internet use. Expert Rev. Neurother. 13(2), 205-213. 